\title{
Plastrum Testudinis Extracts Promote BMSC Proliferation and Osteogenic Differentiation by Regulating Let-7f-5p and the TNFR2/PI3K/AKT Signaling Pathway
}

\author{
Geng-Yang Shen ${ }^{a, c}$ Hui Ren ${ }^{b, c}$ Jin-Jing Huanga, Zhi-Da Zhang ${ }^{a, c}$ \\ Wen-Hua Zhao a,c Xiang Yua,c Qi Shang a,c Ting Qiu ${ }^{a} \quad$ Yu-Zhuo Zhang ${ }^{a}$ \\ Jing-Jing Tang ${ }^{\mathrm{b}, \mathrm{c}}$ De Liang ${ }^{\mathrm{b}, \mathrm{c}}$ Zhi-Dong Yang ${ }^{\mathrm{b}, \mathrm{c}}$ Xiao-Bing Jiang ${ }^{\mathrm{b}, \mathrm{c}}$
}

a'Guangzhou University of Chinese Medicine, Guangzhou, ${ }^{b}$ Department of Spinal Surgery, The First Affiliated Hospital of Guangzhou University of Chinese Medicine, Guangzhou, 'Lingnan Medical Research Center of Guangzhou University of Chinese Medicine, Guangzhou, China

\section{Key Words}

Pte - Let-7f-5p - TNFR2/PI3K/AKT signaling pathway - BMSCs - Proliferation • Osteogenic differentiation

\begin{abstract}
Background/Aims: Plastrum testudinis extracts (PTE) show osteoprotective effects on glucocorticoid-induced osteoporosis in vivo and in vitro. However, the underlying molecular mechanism of PTE in promoting osteogenic differentiation of bone marrow mesenchymal stem cells (BMSCs) is unclear. Methods: BMSC proliferation was investigated using the Cell Counting Kit- 8 assay. BMSC differentiation and osteogenic mineralization were assayed using alkaline phosphatase and Alizarin red staining, respectively. The mRNA expression levels of Let-7f-5p, Tnfr2, Traf2, Pi3k, Akt, $\beta$-catenin, Gsk3 $\beta$, Runx2, and Ocn were measured using real time quantitative polymerase chain reaction. Protein levels of TNFR2, TRAF2, $p$-PI3K, $p$-AKT, $p$ - $\beta$-CATENIN, and p-GSK3 $\beta$ were analyzed by western blotting. The functional relationship of Let-7f-5p and Tnfr2 was determined by luciferase reporter assays. Results: The optimum concentration for PTE was $30 \mu \mathrm{g} / \mathrm{ml}$. PTE significantly promoted BMSC osteogenic differentiation and mineralization after 7 and 14 days in culture, respectively. The combination of PTE and osteogenic induction exhibited significant synergy. PTE upregulated Let-7f-5p, $\beta$-catenin, Runx2, and Ocn mRNA expression, and downregulated Tnfr2, Traf2, Pi3k, Akt, and Gsk3 $\beta$ mRNA expression. PTE inhibited TNFR2, TRAF2, and $p-\beta$-CATENIN protein expression, and promoted $\mathrm{p}$-PI3K, $\mathrm{p}$-AKT, and $\mathrm{p}$-GSK3 $\beta$ protein expression. In addition, Tnfr2 was a functional target of Let-7f-5p in 293T cells. Conclusions: Our results suggested that PTE may promote BMSC proliferation and osteogenic differentiation via a mechanism associated with the regulation of Let-7f-5p and the TNFR2/PI3K/AKT signaling pathway.
\end{abstract}




\section{Introduction}

A delicate balance between bone formation and resorption, also known as bone homeostasis, is required for maintaining the integrity and physiological functions of the skeletal system. Bone marrow mesenchymal stem cells (BMSCs) are common progenitors of osteoblasts and adipocytes in bone, and impaired osteogenic/adipogenic differentiation can result in imbalanced bone homeostasis [1]. Thus, restoring osteogenic differentiation in BMSCs by targeting known regulatory networks is a promising therapeutic strategy to treat patients with various diseases, such as osteoporosis, arthritis, and Paget's disease $[2,3]$. Previously, we showed the protective effect of plastrum testudinis extracts (PTE) in glucocorticoid-induced osteoporosis (GIOP) in rat spine [4, 5]. Moreover, previous studies showed that PTE promoted BMSC proliferation [6.7]. However, the effect of PTE in promoting BMSC osteogenic differentiation remains largely unexplored, and little is known about the downstream mechanisms of PTE-mediated cell signaling.

Tumor necrosis factor (TNF) is a pleiotropic pro-inflammatory cytokine that is both a powerful stimulator of bone resorption and an inhibitor of bone formation [8]. TNF receptor 2 (TNFR2) is a 75-kDa TNF receptor that exhibits a higher affinity for TNF than does TNFR1 [9]. TNFR2 is preferentially activated by membrane-integrated TNF, which leads to the recruitment of TNF receptor-associated factor 2 (TRAF2) to the intracellular TRAF-binding motif. Stimulation of phosphatidylinositol 3-kinase (PI3K)/AKT signaling occurs after TRAF2 recruitment, which then induces IKB kinase (IKK) activation, subsequent

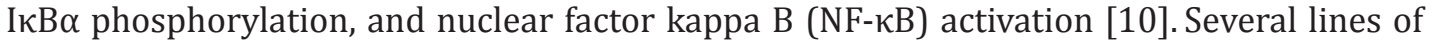
evidence have suggested that the TNFR2 is a strong candidate gene for involvement in peak bone mineral density (BMD) due to its important role in bone turnover and regulation of the effect of TNF on osteoclastogenesis [11]. Additionally, the TNFR2 gene location has been identified as the potential quantitative trait loci for BMD variation in the hip [12]. Significant associations between the TNFR2 gene and BMD variations have been reported in several populations [13-17]. Moreover, in vitro and in vivo studies have suggested that TNFR2 expression is associated with mesenchymal stem cell (MSC) proliferation [18]. Thus, we speculate that TNFR2-mediated TNF signaling pathway plays a pivotal role in regulating BMSC proliferation and osteogenic differentiation.

Micro-RNAs (miRNAs) play important roles as posttranscriptional gene regulators in numerous biological processes including cell development, stem cell regulation, and human diseases. miRNAs are mostly transcribed from intragenic or intergenic regions and undergo further processing by Drosha and other downstream ribonucleases before being converted into mature 18- to 25-nt miRNA transcripts that are capable of gene silencing [19]. Specific miRNAs or miRNA expression profiles are closely involved in controlling the key steps in osteogenic differentiation in BMSCs [20, 21]. Importantly, recent studies in human MSCs (hMSCs) demonstrated that specific Let-7f miRNAs are upregulated during osteogenic differentiation [22, 23]. Moreover, members of the Let- $7 f$ family are thought to function as prodifferentiation factors with "antistemness" properties [24]. For example, mature Let$7 f$ miRNAs are undetectable in embryonic stem cells but show robust accumulation after the onset of differentiation in these cells [25]. However, few studies have focused on the roles of Let-7f in the regulatory networks that control BMSC proliferation and osteogenic differentiation in response to PTE intervention.

In the present study, we showed that PTE acts as a promoter of BMSC proliferation and osteogenic differentiation through positive regulation of Let-7f-5p expression and negative modulation of the TNFR2/PI3K/AKT pathway activity. Furthermore, for the first time, we revealed that the underlying regulatory network involved the Let-7f-5p, as a key factor targeting the translation of Tnfr2. 


\section{Cellular Physiology Cell Physiol Biochem 2018;47:2307-2318

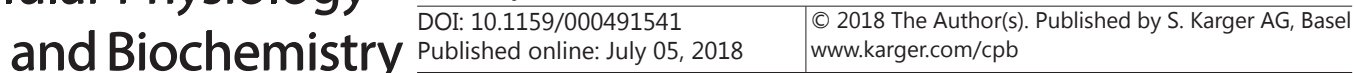 \\ Published online: July 05, 2018}

\section{Materials and Methods}

\section{BMSC isolation and in vitro culture}

BMSCs were obtained from three randomly selected 6-week-old Sprague-Dawley female rats. After euthanasia by sodium pentobarbital and cervical dislocation, bone marrow was flushed out of the femur and tibia with Dulbecco's modified Eagle medium (DMEM; Gibco BRL, Grand Island, NY, USA) supplemented with $10 \%(\mathrm{v} / \mathrm{v})$ fetal bovine serum (FBS; Gibco) and 1\% (v/v) penicillin and streptomycin (Gibco) under standard sterile conditions. The mixture of bone marrow and medium was gently added to the a $50 \%$ Percoll solution (Sigma-Aldrich St. Louis, MO, USA) and centrifuged at 3, $000 \mathrm{rpm}$ for $30 \mathrm{~min}$. The cell suspension was obtained between the layer of Percoll and the supernatant liquid layer. Cells were plated and then incubated in a humidified atmosphere of $5 \% \mathrm{CO}_{2}$ at $37^{\circ} \mathrm{C}$. The cells were passaged every 3 to 4 days using $0.25 \%(\mathrm{w} / \mathrm{v}$ ) trypsin-EDTA solution (Gibco), and used for experiments at the third or fourth passage.

To induce osteoblastic differentiation, the BMSCs were cultured in an osteogenic induction (OI) medium (growth culture medium supplemented with $10^{-8} \mathrm{M}$ dexamethasone) (Sigma-Aldrich), $50 \mu \mathrm{g} / \mathrm{ml}$ ascorbic acid (Sigma-Aldrich), and $5 \mathrm{mM} \beta$-glycerol phosphate (Sigma-Aldrich) at an initial density of $1 \times$ $10^{4}$ cells $/ \mathrm{cm}^{2}$. The medium was changed every 4 days during osteogenesis.

\section{Preparation of PTE}

PTE was obtained according to a method established previously [4-7]. In brief, plastrum testudinis (PT) was purchased from the First Affiliated Hospital of Guangzhou University of Chinese Medicine (Guangzhou, China) and extracted stepwise with ethyl acetate solvent and water; the solvents were recovered to obtain PTE in ethyl acetate, which was dissolved in dimethyl sulphoxide. Gas chromatography-mass spectrometry (GC-MS) and high performance liquid chromatography (HPLC) were performed for determining steroids, fatty acids, and their ester components. This extract contained nine chemical compounds including palmitic acid methyl ester (PubChem CID: 8181), ethyl palmitate (PubChem CID: 12366), methyl stearate (PubChem CID: 8201), stearic acid ethyl ester (PubChem CID: 8122), palmitic acid (PubChem CID: 985), stearic acid (PubChem CID: 5281), cholesterol (PubChem CID: 5997), cholesterol myristate (PubChem CID: 99486), (+)4-cholesten-3-one (PubChem CID: 91477). The concentrations of PTE used in the study were 0 (as control group), $0.03,0.3,3,30$, and $300 \mu \mathrm{g} / \mathrm{mL}$.

\section{Cell proliferation analyses using the Cell Counting Kit (CCK)8 assay}

The proliferation of BMSCs with various concentrations of PTE was measured using the CCK-8 assay (Dojindo Molecular Technologies, Kumamoto, Japan). The BMSCs were cultured in 96-well plates at $1 \times$ $10^{5}$ cells $/ \mathrm{mL}$ with growth culture medium. After $2 \mathrm{~h}$ of cell adhesion, the cells were incubated in media containing various concentrations of PTE for 1,3,5, and 7 days. Next, the BMSCs were seeded in 6-well plates at $1 \times 10^{4}$ cells per well ( 2 wells). After $2 \mathrm{~h}$ of cell adhesion, the cells were incubated in media containing various concentrations of PTE for 14 days. The cells were digested with $0.25 \%$ trypsin. Cells in each well were aspirated with $1 \mathrm{~mL}$ of culture medium, centrifuged at $800 \mathrm{rpm}$ for $5 \mathrm{~min}$, resuspended in $100 \mu \mathrm{L}$ of culture medium, and plated in 96-well plates. On the following day, the CCK8 assay was used to detect cell viability. The remaining wells were cultured for 21 days. Cell viability was measured using the CCK8 assay as described by the manufacturer. The culture fluid was aspirated $4 \mathrm{~h}$ before the end of cell processing. The CCK- 8 assay was performed by incubating in $10 \%$ CCK-8 solution at $37^{\circ} \mathrm{C}$ in a $5 \% \mathrm{CO}_{2}$ incubator for $4 \mathrm{~h}$. For CCK-8 measurement, $100 \mu \mathrm{L}$ of the incubated cell suspension was transferred to a 96-well plate. The absorbance of the culture medium was measured at $450 \mathrm{~nm}$ using a microplate reader. The optimal concentration of PTE was selected for inducing osteogenic differentiation according to the results of the CCK8 assay. The assay groups were treated as follows: (1) the control group received FBS medium, (2) the PTE group received FBS medium with PTE, (3) the OI group received OI medium, and (4) the PTE+OI group received OI medium with PTE.

\section{Alkaline phosphatase (ALP) activity assays}

For ALP staining, BMSCs were cultured in 24-well plates at a cell density of $1 \times 10^{4}$ and analyzed on day 21 using an ALP staining kit (Beyotime, Shanghai, China). Cells that were cultured in OI medium were rinsed twice with phosphate-buffered saline (PBS) and fixed with 4\% paraformaldehyde. Briefly, after formalin fixation for $10 \mathrm{~min}$, 5-bromo-4-chloro-3-indolyl phosphate (BCIP)/nitro blue tetrazolium (NBT) working 


\section{Cellular Physiology Cell Physiol Biochem 2018;47:2307-2318 \begin{tabular}{l|l|l} 
and Biochemistry.1159/000491541 & $\begin{array}{l}\text { ( ) 2018 The Author(s). Published by S. Karger AG, Basel } \\
\text { www.karger.com/cpb }\end{array}$
\end{tabular}}

Shen et al.: PTE Promote BMSC Osteogenic Differentiation

solution was added to each well and incubated in the dark at room temperature for $30 \mathrm{~min}$. The cells were rinsed twice with distilled water, counterstained with Neutral red (Beyotime) for $5 \mathrm{~min}$, and photographed under a microscope.

ALP activity was determined using an ALP assay kit in 6-well plates at a cell density of $1 \times 10^{4}$. The cells were rinsed three times with cold PBS and lysed in radioimmunoprecipitation assay buffer (RIPA; Sigma-Aldrich) on ice. The total protein concentration was determined using a BCA protein assay kit (Beyotime). The optical density (OD) of the ALP protein was measured at $520 \mathrm{~nm}$ according to the manufacturer' $\mathrm{s}$ instruction. ALP activity was calculated using the ALP level normalized to the total protein.

\section{Alizarin red staining}

BMSCs were seeded at $1 \times 10^{4}$ cells per
Table 1. Primers used for quantitative RT-qPCR

\begin{tabular}{ll}
\hline ID & Sequence (5'-3') \\
\hline Let-7f-5p & TGAGGTAGTAGATTGTATAGTT \\
Let-7f-5p-RT & CTCAACTGGTGTCGTGGAGTCGGCAATTCAGTTGAGAACTATA \\
Let-7f-5p-F & ACACTCCAGCTGGGTGAGGTAGTAGATTGTAT \\
Tnfr2-F & TCAGCCAGAGCCCACAAGA \\
Tnfr2-R & GAAGCAGTTCGCCAGTCCTA \\
Traf2-F & TGAAAGGTCCCAATGATGC \\
Traf2-R & GGAGGACGAAGTTACATCAGG \\
Pi3k-F & ACGGCAATGTGGAGCAGA \\
Pi3k-R & GTCGTAGCCAATCAGGGAG \\
Akt-F & CTTTATTGGCTACAAGGAACGG \\
Akt-R & CAGTCTGAATGGCGGTGGT \\
$\beta-$-catenin-F & TGCAGCGACTAAGCAGGA \\
$\beta-$ catenin-R & TCACCAGCACGAAGGACA \\
Gsk3 $\beta-F$ & GTCCGATTGCGGTATTTCT \\
Gsk3 $\beta-R$ & ACAGGGAGTGTCTGCTTGG \\
Runx2-F & GACTGTGGTTACCGTCATGGC \\
Runx2-R & ACTTGGTTTTTCATAACAGCGGA \\
Ocn-F & CTGACCTCACAGATCCCAAGC \\
Ocn-R & TGGTCTGATAGCTCGTCACAAG \\
$\beta$-actin-F & GGAGATTACTGCCCTGGCTCCTA \\
$\beta$-actin-R & GACTCATCGTACTCCTGCTTGCTG \\
\hline
\end{tabular}
well onto 24-well plates, and the medium was changed after $24 \mathrm{~h}$ of incubation. Cells in the various groups were fixed with $4 \%$ parafomadelyde for 15 min at $37^{\circ} \mathrm{C}$ and rinsed three times with distilled water. Then, the fixed cells were stained for 15 min in Alizarin red solution (1.0 g Alizarin powder was dissolved into $50 \mathrm{~mL}$ distilled water and adjusted to $\mathrm{pH} 4.2$ ). Finally, the cells were washed in distilled water to remove excess stain and observed using a microscope. Quantitation was achieved by dissolving the mineralized nodules with hexadecylpyridinium chloride, and the absorbance (OD value) of the liquid was measured at $578 \mathrm{~nm}$.

\section{Real time quantitative polymerase chain reaction (RT-qPCR) analyses}

BMSCs were harvested, and total RNA was extracted using the MiniBEST Universal RNA Extraction Kit (Cat. No. 9767; Takara Bio Inc., Japan) according to the manufacturer's protocol. Reverse transcription was performed using $60 \mathrm{uL}$ of reaction solution (up to $500 \mathrm{ng}$ total RNA was contained in $10 \mu \mathrm{L}$ of reaction solution) with the PrimeScript RT Master Mix kit (Perfect Real Time, Cat No. RR036A; Takara). Real-time analyses were performed using the SYBRR Premix Ex TaqTMII kit (TilRNaseH Plus, Cat \# RR820A; TaKaRa) on a iQ5 PCR cycler (Bio-Rad, Hercules, CA, USA). A total of 2 uL cDNA was amplified using the primers listed in Table 1. The genes assayed comprised Let-7f-5p, Tnfr2, Traf2, Pi3k, Akt, $\beta$-catenin, Gsk3 $\beta$, Runx2, and Ocn. The RT-qPCR parameters were as follows: $50^{\circ} \mathrm{C}$ for $2 \mathrm{~min}$ and $95^{\circ} \mathrm{C}$ for $10 \mathrm{~min}$, followed by 40 cycles of $95^{\circ} \mathrm{C}$ for $15 \mathrm{~s}$ and $60^{\circ} \mathrm{C}$ for $60 \mathrm{~s}$. $\beta$-actin was used as an internal control, and all experiments were performed in triplicate. Relative expression levels of each gene were quantified by using the $2^{-\mathrm{D} \Delta \mathrm{Ct}}$ method.

\section{Western blot analyses}

BMSCs were harvested and lysed using RIPA buffer (Beyotime). Protein levels in the supernatant were determined using the BCA Protein Assay Kit (Tiangen Biotech Co., Ltd., Beijing, China). Equal amounts of proteins $(20 \mu \mathrm{g})$ were separated on 6-18\% sodium dodecyl sulfate polyacrylamide gel electrophoresis and transferred to polyvinylidene difluoride membranes (Millipore, Billerica, MA, USA). The membranes were blocked with $5 \%$ skim milk for $1 \mathrm{~h}$ and washed three times with TBST. The membranes were incubated with a primary antibody against TNFR2 (\#3727; 1:1, 000; CST, Framingham, MA USA), TRAF2 (\#4712; 1:1, 000; CST), p-PI3K (ab182651; 1:1, 000; Abcam, Cambridge, UK), p-AKT (\#4060; 1:2, 000; CST), p- $\beta$-CATENIN (\#9561; 1:1, 000; CST), p-GSK3ß (\#5558; 1:1, 000; CST), and GAPDH (RC-5G5; 1:10, 000; KangChen Biotech, Shanghai, China), overnight at $4^{\circ} \mathrm{C}$. The membranes were incubated with horseradish peroxidaseconjugated secondary antibodies for $1 \mathrm{~h}$ at room temperature. Protein expression signals were visualized by enhanced chemiluminescence (ECL; Beyotime). And band intensities were quantified using Image J software (National Institutes of Health, Bethesda, MD, USA).

\section{Plasmid construction}

Let-7f-5p mimics and negative control (NC) mimics were synthesized by Biomics Biotech Co., Ltd. (Nantong, China). To generate the luciferase reporter vector, a 220-bp fragment of the Tnfrsf1b (Tnfr2) 
mRNA 3' untranslated region (UTR) containing the predicted Let-7f-5p binding site (position 794-801) (Tnfr2 mRNA 3' UTR-WT) and its mutant sequence (Tnfr2 mRNA 3' UTR-MUT) were synthesized by Genechem and cloned into psoCHECK2 vectors (Promega Biotech Co., Ltd., Beijing, China). The sequences of all constructs were confirmed by DNA sequencing. The primers used are listed in Table 2.
Table 2. Primers luciferase reporter constructs

\begin{tabular}{ll}
\hline ID & Sequence $\left(5^{\prime}-3^{\prime}\right)$ \\
\hline Tnfrsf1b-MUT-F & GCCACCTGTCTCGTCAATGGCTTGCCTGGAGCACTCTG \\
Tnfrsf1b-MUT-R & CAGAGTGCTCCAGGCAAGCCATTGACGAGACAGGTGGC \\
Tnfrsf1b-XhoI-F & CCGCTCGAGTTTATCCTCTGCCTTTGACTTTTACTCC \\
Tnfrsf1b-NotI-R & ATTGCGGCCGCATTAGAAAATAAGCATGAATCCTTTGGC \\
\hline
\end{tabular}

\section{Transfection}

For transient transfections, a $20 \mathrm{nM}$ final concentration of Let-7f-5p mimics, NC mimics, Tnfr2 3' UTRWT, and Tnfr2 3' UTR-MUT in Opti-MEM (Invitrogen, Carlsbad, CA, USA) was combined with Lipofectamine 2000 (Invitrogen) in serum-free medium and incubated at room temperature for $20 \mathrm{~min}$. The mixture was then added to $293 \mathrm{~T}$ cells in 24- or 6-well plates at $80 \%$ fusion. The medium was replaced by proliferation or differentiation medium $6 \mathrm{~h}$ later. For long-term detection under differentiation culture conditions, the cells were transfected every 3 days.

\section{Luciferase reporter assays}

The 293T cells were transfected with either the Let-7f-5p mimics or NC mimics in conjunction with the luciferase reporter constructs. At $48 \mathrm{~h}$ post-transfection, the cells were lysed and subjected to luciferase assays using a Dual Luciferase Reporter Assay System (Promega) according to the manufacturer' s protocol. The assay was performed in triplicate.

\section{Statistical analysis}

The data were analyzed with SPSS software (ver. 19.0; SPSS Inc., Chicago, IL, USA). For quantitative analysis, all results are expressed as mean \pm standard deviations (SD). Statistical significance was evaluated by one-way analysis of variance (ANOVA) with the Student-Newman-Keuls (SNK) test applied for post hoc analysis. Two-sided $P$-values $<0.05$ were considered to indicate statistical significant.

\section{Results}

PTE promoted BMSC proliferation.

BMSC proliferation was analyzed on days 1, 3, 5, 7, 14, and 21 days (Fig. 1). The relative cell number (OD value) at each concentration of PTE increased gradually in a time-dependent manner until day 7. Greater numbers of BMSCs were observed with treatment by PTE at concentration of $0.03,0.3,3,30$, and $300 \mu \mathrm{g} / \mathrm{mL}$ compared with control-treated BMSCs $(0$ $\mu \mathrm{g} / \mathrm{mL}$ ); however, the differences showed no statistical significance. After 7 days, a dosedependent decrease in the quantity of BMSCs was observed at each concentration of PTE. BMSC proliferation was lowest in the absence of PTE $(0 \mu \mathrm{g} / \mathrm{mL})$ and highest in the presence of $300 \mu \mathrm{g} / \mathrm{mL}$ PTE $(P<0.05)$; however, no significant difference was observed compared with that at the PTE concentration of $30 \mu \mathrm{g} / \mathrm{mL}$. Furthermore, BMSC proliferation showed a steady and slight increase on day 14 when treated with PTE at a concentration of $30 \mu \mathrm{g} / \mathrm{mL}$.

\section{PTE promoted BMSC osteogenic differentiation}

We compared the early differentiation of BMSCs after treatment with PTE, OI medium, and the combination of PTE and OI medium by performing ALP staining on days 3, 7, 14, and 21 (Fig. 2A). BMSCs cultured for 7 days in OI group and PTE+OI group showed stronger ALP staining intensity than those in the control and PTE groups. Moreover, after 14 and 21 days in culture, the PTE, OI, and PTE+OI groups showed significantly stronger ALP staining intensity compared with the control group. ALP staining in the PTE+OI group showed the strongest intensity at each time point. Quantitative ALP assays further confirmed the results of ALP staining (Fig. 2B).

We compared the osteogenic mineralization ability of the BMSCs after treatment with PTE, OI medium, and the combination of PTE and OI medium by performing Alizarin red 
Fig. 1. Effect of PTE on BMSC proliferation was analyzed on day $1,3,5,7,14$, and 21 . The experiment was repeated for 3 times, and the data were expressed as mean $\pm \mathrm{SD}^{*} \mathrm{p}<0.05$ versus the control group. The OD 450 values were measured at the specific time points to draw curves.

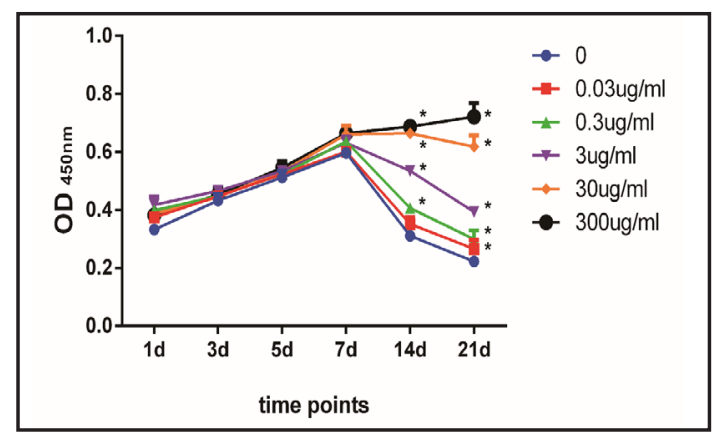

Fig. 2. BMSC osteogenic differentiation assay. A. the ALP staining assay of BMSC treated with PTE, OI medium, and the combination of PTE and OI medium for 3, 7, 14, 21 days. Scale bar, $100 \mu \mathrm{m}$. B. Quantitative assay of ALP activity at day 3, 7, 14, and 21 . The experiment was repeated for 3 times, and the data were expressed as mean $\pm \mathrm{SD}^{*} \mathrm{p}<0.05$ versus the control group, ${ }^{\#} \mathrm{p}<0.05$ versus the PTE group, ${ }^{\Delta} \mathrm{p}<0.05$ versus the OI group.

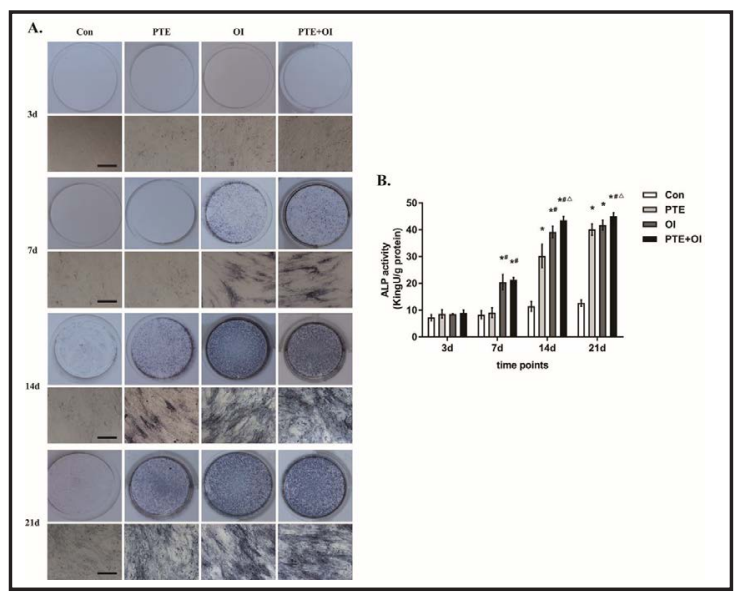

Fig. 3. BMSC osteogenic mineralization assay. A. Alizarin red staining of BMSCs treated with PTE, OI medium, and the combination of PTE and OI medium for 3, 7, 14, 21 days. Scale bar, $100 \mu \mathrm{m}$. B. Quantitative assay of Alizarin red staining. The experiment was repeated for 3 times, and the data were expressed as mean $\pm \mathrm{SD} * \mathrm{p}<0.05$ versus the control group, ${ }^{\mathrm{p}}<0.05$ versus the PTE group, ${ }^{\Delta} \mathrm{p}<0.05$ versus the OI group.

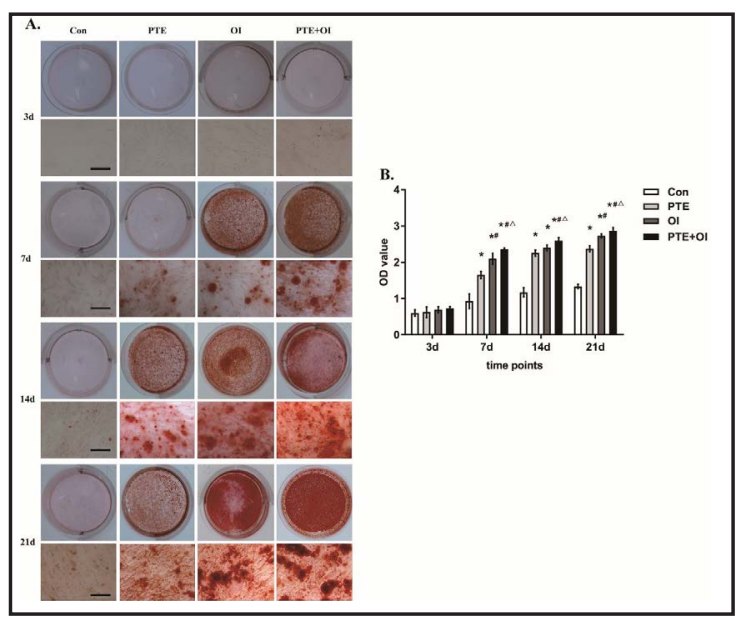

staining on days 3, 7, 14, and 21 (Fig. 3A). BMSCs cultured for 7, 14, and 21 days formed mineralized nodules in the PTE, OI, and PTE+OI group; the amount of matrix mineralization was highest in the PTE+OI group at each time point. Quantitative measurement of Alizarin red staining further confirmed the results (Fig. 3B).

PTE upregulated Let-7f-5p, $\beta$-catenin, Runx2, and Ocn mRNA expression, and downregulated Tnfr2, Traf2, Pi3k, Akt, and Gsk3 $\beta$ mRNA expression during BMSC osteogenic differentiation

We investigated the mRNA expression levels of Let-7f-5p, four target genes of the TNFR2/PI3K/AKT signaling pathway (Tnfr2, Traf2, Pi3k, and Akt), $\beta$-catenin, Gsk3 $\beta$, Runx2, and Ocn after 21 days in culture during osteogenic differentiation (Fig. 4). The PTE, OI, and PTE+OI groups showed significant upregulation of Let-7f-5p, $\beta$-catenin, Runx2, and Ocn mRNA expression, but significant downregulation of Tnfr2, Traf2, Pi3k, Akt, and Gsk3 $\beta$ 
mRNA expression. No differences were detected in the mRNA expressions of Thfr2, Traf2, $A k t, \beta$-catenin, and Gsk3 $\beta$ between the PTE and OI groups. However, the OI group showed significantly lower levels of Let-7f-5p and Pi3k mRNA compared with the PTE group. Furthermore, the PTE+OI group showed the highest levels of Let-7f-5p and $\beta$-catenin mRNA, and the lowest levels of Tnfr2 and Traf2 mRNA among the experimental groups.

PTE inhibited TNFR2, TRAF2, and $p$ - $\beta$-CATENIN protein expression, and promoted $p$-PI3K, $p$-AKT, and $p$-GSK3 $\beta$ protein expression during BMSC osteogenic differentiation

Western blot assays were performed to investigate the protein expression of TNFR2, TRAF2, p-PI3K, p-AKT, p- $\beta$-CATENIN, and p-GSK3 $\beta$ after 21 days in culture during OI (Fig. 5 ). Compared with the control group, the PTE, OI, and PTE+OI groups exhibited significantly lower protein expression levels of TNFR2, TRAF2, and p- $\beta$-CATENIN, but significantly higher levels of p-PI3K and p-GSK3 $\beta$. Additionally, no differences in TNFR2, p-PI3K, or p- $\beta$-CATENIN protein expression levels were detected between the PTE and OI groups. Nevertheless, the OI group showed significantly higher protein expression levels of TRAF2, but significantly lower levels of p-AKT and p-GSK3 $\beta$ compared with the PTE group. Moreover, the PTE+OI group exhibited the lowest protein expression levels of TNFR2, TRAF2, and p- $\beta$-CATENIN, but the highest levels of p-PI3K, p-AKT, and p-GSK3 $\beta$ among the experimental groups.

\section{Let-7f-5p negatively regulated TNFR2 in $293 T$ cells}

miRNAs modulate cell proliferation and differentiation by downregulating the expression of downstream targets. Mechanistically, miRNAs bind to the 3' UTR of mRNAs, which leads

Fig. 4. The relative mRNA expression levels of Let-7f-5p, four target genes of the TNFR2/ PI3K/AKT signaling pathway (Tnfr2, Traf2, Pi3k, and Akt), $\beta$-catenin, Gsk3 $\beta$, Runx2, and Ocn were showed. The experiment was repeated for 3 times, and the data were expressed as mean \pm SD ${ }^{*} \mathrm{p}<0.05$ versus the control group, $\# p<0.05$ versus the PTE group, ${ }^{\Delta} \mathrm{p}<0.05$ versus the OI group.
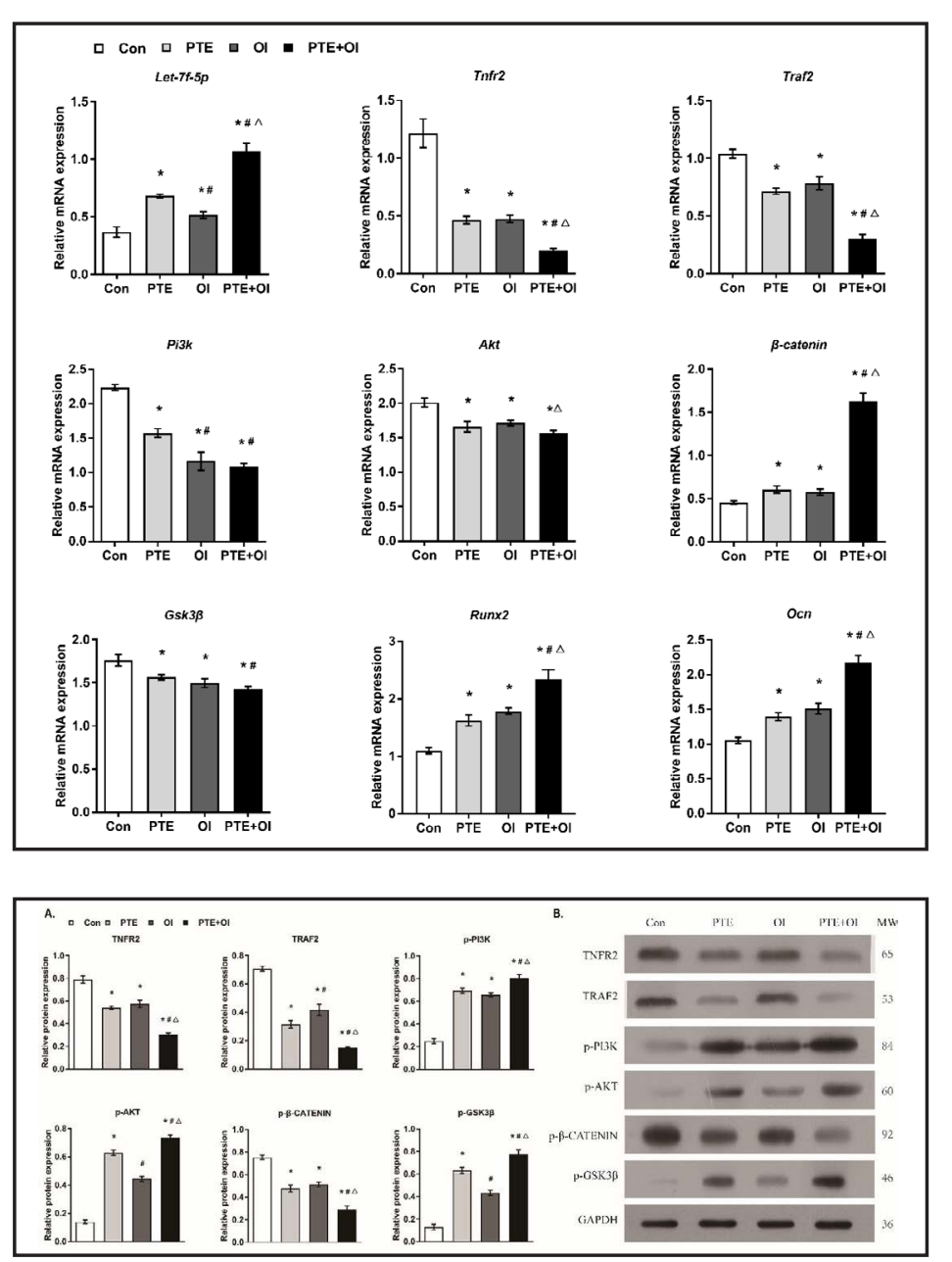

Fig. 5. Protein expression levels of TNFR2, TRAF2, p-PI3K, p-AKT, $p$ - $\beta$-CATENIN, and $p$-GSK3 $\beta$ were analyzed by Western blot. The experiment was repeated for 3 times, and the data were expressed as mean $\pm \mathrm{SD} * \mathrm{p}<0.05$ versus the control group, $" \mathrm{p}<0.05$ versus the PTE group, ${ }^{\Delta} \mathrm{p}<0.05$ versus the OI group. 
to consequential mRNA degradation or inhibition of translation. Using the online program TargetScan (data not shown), we identified several potential targets of Let-7f-5p including Tnfr2, an established transcription factor and main target of Let-7f-5p. We targeted the Let-7f-5p binding sites at 794-801 of the Tnfr2 3' UTR (Fig. 6A) and constructed plasmids containing wild type and mutant Tnfr2 to transfect 293T cells. Luciferase reporter assays were performed after $48 \mathrm{~h}$ transfection. The luciferase reporter assays showed significantly lower luciferase activity in the Let-7f-5p and Tnfr2 cotransfected cells (Fig. 6B), indicating that Let-7f-5p bound to the 3' UTR of Tnfr2. The complementary binding of the miRNA to the 3' UTR mRNA resulted in degradation of the target gene.

\section{Discussion}

This study investigated the mechanism involved in PTE regulation of BMSC proliferation and osteogenic differentiation. PTE stimulated BMSC proliferation in a time- and dose-

Fig. 6. Let- $7 f-5 p$ negatively regulates $\mathrm{Tnfr} 2$ by binding to the $\mathrm{Tnfr} 2$ 3' UTR. A. Schematic of putative Let-7f-5p binding sites and mutated binding sites in Tnfr2 3' UTR. Putative Let-7f-5p binding site and mutated binding sites was indicted with red character. B. Luciferase reporter assay of $293 \mathrm{~T}$ cells. Column, means; bar, SD. ${ }^{* * *} \mathrm{P}<0.01$ vs negative control (NC) mimics.

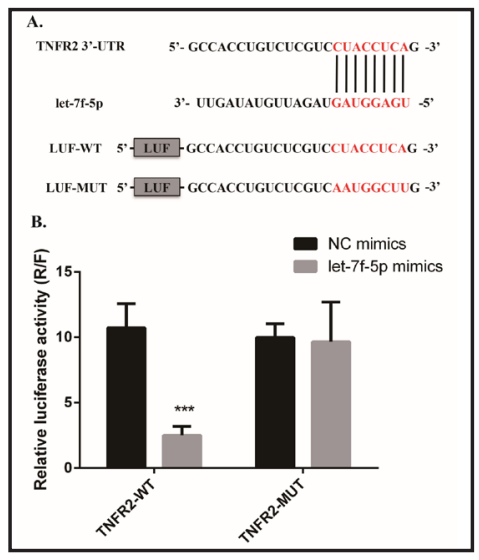

Fig. 7. The flow chart on the action mechanism of PTE. Differential expression levels of miRNAs cluster (heat map) analyses and volcanoplot of vertebral samples of glucocorticoidinduced osteoporosis (GIOP) by performing miRNA-seq analysis and qRT-PCR validation and Let$7 f-5 p$ was selected. PTE may promote BMSC proliferation and osteogenic differentiation, and the mechanism may be associated with the regulation of Let-7f-5p and the TNFR2/PI3K/AKT signaling pathway. " $\uparrow$ " represents promoting effect and " $\mathrm{T}$ " represents inhibiting effect.

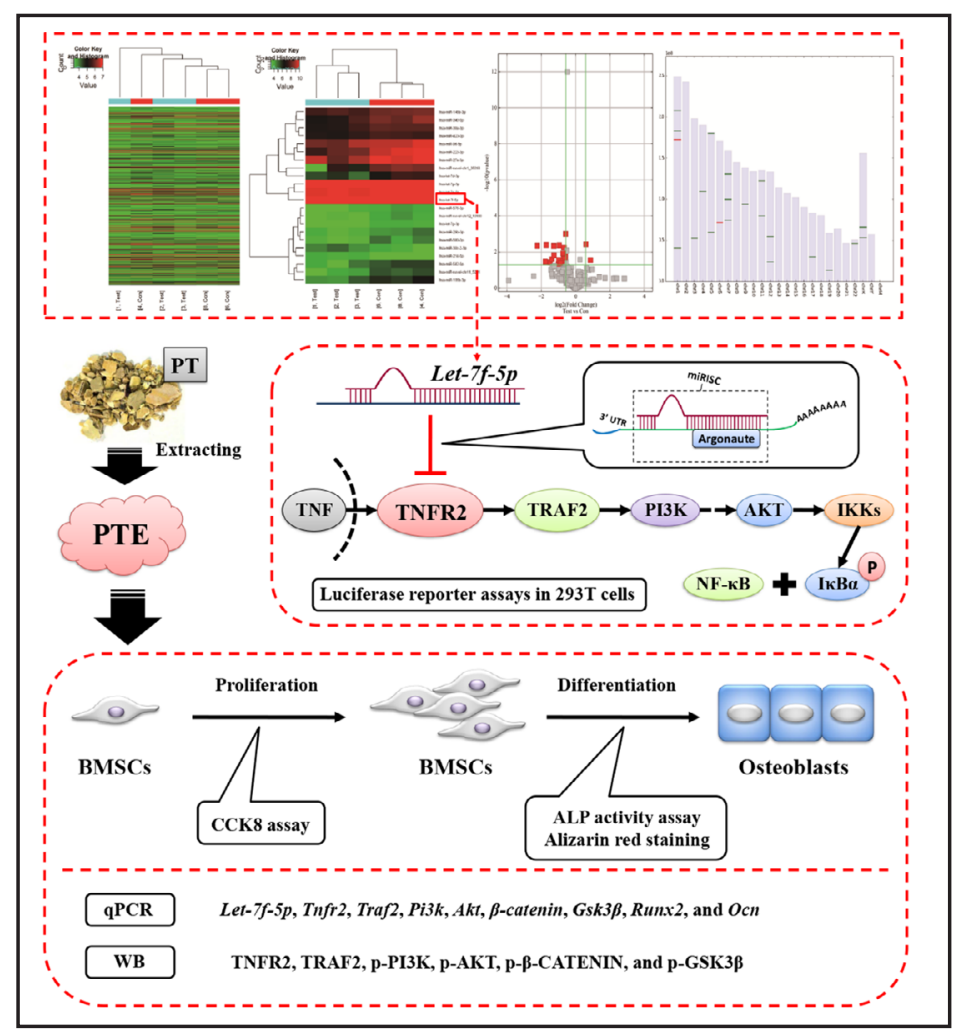


dependent manner, which peaked at day 7. This result was consistent with that described in a previous study, which showed that PTE treatment for 4 days induced cell proliferation [26]. Moreover, PTE treatment at a concentration of $30 \mu \mathrm{g} / \mathrm{mL}$ slightly and consistently promoted BMSC proliferation from day 7 to 14 . This result indicated that the PTE concentration of 30 $\mu \mathrm{g} / \mathrm{mL}$ was the optimum concentration, in accordance with a previous study indicating day 14 as the optimal treatment time [6]. However, to the best of our knowledge, no previous studies have reported the role of PTE in regulating BMSC osteogenic differentiation.

ALP and Alizarin red staining showed that treatment with PTE, OI, and PTE+OI significantly promoted BMSC osteogenic differentiation, suggesting synergistic effects of ALP and OI. Moreover, the effect of PTE in promoting BMSC osteogenic differentiation was further verified by the presence of increased mRNA expressions of $\beta$-catenin, Gsk3 $\beta$, Runx 2 , and Ocn, and increased protein expression of p- $\beta$-CATENIN and p-GSK3 $\beta$, which are important in the Wnt/ $\beta$-catenin signaling pathway [27]. Therefore, PTE may be a promising therapeutic agent against a series of bone metabolic diseases associated with impaired BMSC osteogenic differentiation. This in vitro study may shed light on the effect of PTE in rescuing glucocorticoid-induced spinal osteoporosis in rats $[4,5]$.

Although previous studies suggested the importance of TNFR2 in regulating osteoclastogenesis, peak BMD, and MSC proliferation, the potential mechanism underlying the TNFR2-mediated TNF signaling pathway in BMSC proliferation and osteogenic differentiation requires further elucidation. TRAF2 recruitment is a key step in the TNFR2mediated TNF- $\alpha$ signal transduction pathway. A dominant-negative mutant of TRAF2 blocks TNFR2-mediated NF- $\kappa$ B activation, whereas overexpression of TRAF2 causes activation of $\mathrm{NF}-\kappa \mathrm{B}$, suggesting that TRAF2 plays a pivotal role in TNFR2-mediated NF- $\kappa \mathrm{B}$ activation [26]. In addition, PI3K/AKT signaling acts as a central regulator in bone tissue metabolism and proliferation, and osteogenic differentiation of MSCs [28-31]. The present study revealed for the first time that PTE downregulates Tnfr2, Traf2, Pi3k, and Akt mRNA expression, and promotes p-PI3K, p-AKT, and p-GSK3 $\beta$ protein expression during BMSC osteogenic differentiation, demonstrating that the ability of PTE to stimulate osteogenic differentiation in BMSCs is dependent on the TNFR2/PI3K/AKT signaling pathway. Recently, TNFR2 was identified as a key drug target for cancer immunotherapy [32] and various autoimmune diseases [18], suggesting that the TNFR2-mediated TNF signaling pathway may be involved in regulating the immune system and inflammation. Interestingly, along with PTE-induced BMSC osteogenic differentiation, proinflammatory signaling cascades are suppressed by PTE, suggesting that anti-inflammatory therapies may be applicable for treating bone mass loss, which is associated with impaired BMSC osteogenic differentiation [33]. Therefore, PTE has the potential to be an effective anti-inflammatory drug.

To further elucidate the intracellular molecular mechanism by which PTE promotes BMSC proliferation and osteogenic differentiation, we focused on miRNAs, which play important roles in the functionality of BMSCs [20,21]. The expression of miR-20a is elevated during osteogenic differentiation, and overexpression of miR-20a promotes hMSC osteogenic differentiation by targeting PPAR $\gamma, \mathrm{BAMBI}$, and CRIM1 [34]. miR-194 regulates MSC osteogenic differentiation, but inhibites adipocyte differentiation by directly targeting COUP-TFII [35]. In addition, by exerting post-transcriptional control of their specific target genes, miRNAs could regulate osteogenesis through multiple signaling pathways, such as bone morphogenic protein, Notch, Wnt, and transforming growth factor- $\beta$ signaling pathways [21]. In our previous study, we used vertebral samples of GIOP to perform miRNAseq analysis and qRT-PCR validation. Using this strategy, we identified nine differentially expressed miRNAs, of which six (hsa-mir-186-5p, hsa-mir-21-5p, hsa-mir-214-5p, hsa-mir10b-5p, hsa-mir-451a, and hsa-mir-novel-chr3_49413) were significantly upregulated and three (hsa-let-7f-5p, hsa-let-7a-5p, and hsa-mir-27a-3p) were significantly downregulated (Fig. 7). Additionally, the important role of Let-7f in osteogenic differentiation has been reported in previous studies [22-25], so we continuously selected Let-7f to explore the underlying molecular mechanism of PTE in promoting BMSC osteogenic differentiation. For the first time, the present study showed that the expression of Let-7f-5p is significantly 
elevated during BMSC osteogenic differentiation induced by PTE, OI, and PTE+OI. Our luciferase assays results further confirmed that Let-7f-5p directly targeted the 3' UTR of Tnfr2 in 293T cells (Fig. 7). This study was limited in that we did not investigate whether knockdown or knockout of Let-7f-5p affects the TNFR2/PI3K/AKT signaling pathway or proliferation/osteogenic differentiation in PTE-treated BMSCs, in vitro or in vivo. Further studies investigating the molecular mechanism will be required to reveal the relationships among Let-7f-5p, TNFR2/PI3K/AKT and BMSC osteogenic differentiation.

In this study, we demonstrated that PTE is a positive regulator of BMSC proliferation and osteogenic differentiation. The underlying mechanism involves the upregulation of Let$7 f-5 p$ miRNA expression levels and inhibition of the signal transduction of the TNFR2/PI3K/ AKT pathway. Importantly, our results showed a direct relationship between Let-7f-5p and Tnfr2, suggesting that PTE may promote BMSC proliferation and osteogenic differentiation. The mechanism of action may be associated with the regulation of Let-7f-5p and the TNFR2/ PI3K/AKT signaling pathway. A deeper knowledge of this regulatory network may lead to the development of novel therapeutic approaches to enhance bone formation during pathological bone loss.

\section{Acknowledgements}

The present study was funded by the National Natural Science Foundation of China [grant numbers 81503591 and 81774338 to Xiao-Bing Jiang; 81674000 to De Liang], Guangdong Science and Technology Department (grant numbers 2014A020221021 to Xiao-Bing Jiang, 2016A020226006 to De Liang), Guangdong Natural Science Foundation (grant numbers 2014A030310082 to Xiao-Bing Jiang, 2016A030313645 to Zhi-Dong Yang), Excellent Doctor Project of the First School of Clinic Medicine of Guangzhou University of Chinese Medicine (grant numbers YB201602 to Geng-Yang Shen, YB201501 to Hui Ren), Projects of Traditional Chinese Medicine Bureau of Guangdong Province (grant numbers 20161136 to Jing-Jing Tang) and Excellent Young Scholars Project of the First Affiliated Hospital of Guangzhou University of Chinese Medicine (grant numbers 2015QN03 to Jing-Jing Tang).

We thank The National Key Discipline and The Orthopaedic Laboratory of Guangzhou University of Chinese Medicine for providing associated facilities. We also thank Prof. Dongfeng Chen for providing the basis associated with extracts from plastrum testudinis.

\section{Disclosure Statement}

The authors declare to have no conflict of interests.

\section{References}

1 Crane JL, Cao X: Bone marrow mesenchymal stem cells and TGF-beta signaling in bone remodeling. J Clin Invest 2014;124:466-472.

-2 Baum R, Gravallese EM: Impact of inflammation on the osteoblast in rheumatic diseases. Curr Osteoporos Rep 2014;12:9-16.

3 Rachner TD, Khosla S, Hofbauer LC: Osteoporosis: now and the future. Lancet 2011;377:1276-1287.

4 Liang D, Ren H, Qiu T, Shen G, Xie B, Wei Q, Yao Z, Tang J, Zhang Z, Jiang X: Extracts from plastrum testudinis reverse glucocorticoid-induced spinal osteoporosis of rats via targeting osteoblastic and osteoclastic markers. Biomed Pharmacother 2016;82:151-160.

5 Ren H, Shen G, Tang J, Qiu T, Zhang Z, Zhao W, Yu X, Huang J, Liang D, Yao Z, Yang Z, Jiang X: Promotion effect of extracts from plastrum testudinis on alendronate against glucocorticoid-induced osteoporosis in rat spine. Sci Rep 2017;7:10617. 


\section{Cellular Physiology Cell Physiol Biochem 2018;47:2307-2318 \begin{tabular}{ll|l} 
and Biochemistry & $\begin{array}{l}\text { DOI: 10.1159/000491541 } \\
\text { Published online: July 05, } 2018\end{array}$ & $\begin{array}{l}\text { @ } 2018 \text { The Author(s). Published by S. Karger AG, Basel } \\
\text { www.karger.com/cpb }\end{array}$ \\
\hline
\end{tabular}}

-6 Chen DF, Zeng HP, Du SH, Li H, Zhou JH, Li YW, Wang TT, Hua ZC: Extracts from Plastrum testudinis promote proliferation of ratbone-marrow-derived mesenchymal stem cells. Cell Prolif 2007;40:196-212.

7 Wang TT, Chen W, Zeng HP, Chen DF: Chemical components in extracts from Plastrum testudinis with proliferation-promoting effects on rat mesenchymal stem cells. Chem Biol Drug Des 2012;79:1049-1055. Williams A, Wang EC, Thurner L, Liu CJ: Review: Novel Insights Into Tumor Necrosis Factor Receptor, Death Receptor 3, and Progranulin Pathways in Arthritis and Bone Remodeling. Arthritis Rheumatol 2016;68:2845-2856.

-9 Tartaglia LA, Goeddel DV: Two TNF receptors. Immunol Today 1992;13:151-153.

10 Naude PJ, den Boer JA, Luiten PG, Eisel UL: Tumor necrosis factor receptor cross-talk. FEBS J 2011;278:888-898.

11 Abu-Amer Y, Erdmann J, Alexopoulou L, Kollias G, Ross FP, Teitelbaum SL: Tumor necrosis factor receptors types 1 and 2 differentially regulate osteoclastogenesis. J Biol Chem 2000;275:27307-27310.

-12 Devoto M, Specchia C, Li HH, Caminis J, Tenenhouse A, Rodriguez H, Spotila LD: Variance component linkage analysis indicates a QTL for femoral neck bone mineral density on chromosome 1p36 Hum Mol Genet 2001;10:2447-2452.

13 Spotila LD, Rodriguez H, Koch M, Adams K, Caminis J, Tenenhouse HS, Tenenhouse A: Association of a polymorphism in the TNFR2 gene with low bone mineral density. J Bone Miner Res 2000;15:1376-1383.

14 Spotila LD, Rodriguez H, Koch M, Tenenhouse HS, Tenenhouse A, Li H, Devoto M: Association analysis of bone mineral density and single nucleotide polymorphisms in two candidate genes on chromosome 1p36 Calcif Tissue Int 2003;73:140-146.

15 Albagha OM, Tasker PN, McGuigan FE, Reid DM, Ralston SH: Linkage disequilibrium between polymorphisms in the human TNFRSF1B gene and their association with bone mass in perimenopausal women. Hum Mol Genet 2002;11:2289-2295.

-16 Tasker PN, Albagha OM, Masson CB, Reid DM, Ralston SH: Association between TNFRSF1B polymorphisms and bone mineral density, bone loss and fracture. Osteoporos Int 2004;15:903-908.

17 Xu H, Zhao LJ, Lei SF, Li MX, Sun X, Deng FY, Jiang DK, Deng HW: The (CA)n polymorphism of the TNFR2 gene is associated with peak bone density in Chinese nuclear families. J Hum Genet 2005;50:301-4.

18 Faustman D, Davis M: TNF receptor 2 pathway: drug target for autoimmune diseases. Nat Rev Drug Discov 2010;9:482-493.

19 Adams BD, Parsons C, Walker L, Zhang WC, Slack FJ: Targeting noncoding RNAs in disease. J Clin Invest 2017;127:761-771.

20 Shen G, Ren H, Qiu T, Liang D, Xie B, Zhang Z, Yao Z, Yang Z, Jiang X: Implications of the Interaction Between miRNAs and Autophagy in Osteoporosis. Calcif Tissue Int 2016;99:1-12.

21 Huang C, Geng J, Jiang S: MicroRNAs in regulation of osteogenic differentiation of mesenchymal stem cells. Cell Tissue Res 2017;368:229-238.

22 Oskowitz AZ, Lu J, Penfornis P, Ylostalo J, McBride J, Flemington EK, Prockop DJ, Pochampally R: Human multipotent stromal cells from bone marrow and microRNA: regulation of differentiation and leukemia inhibitory factor expression. Proc Natl Acad Sci U S A 2008;105:18372-18377.

23 Goff LA, Boucher S, Ricupero CL, Fenstermacher S, Swerdel M, Chase LG, Adams CC, Chesnut J, Lakshmipathy U, Hart RP: Differentiating human multipotent mesenchymal stromal cells regulate microRNAs: prediction of microRNA regulation by PDGF during osteogenesis. Exp Hematol 2008;36:13541369.

24 Peter ME: Let-7 and miR-200 microRNAs: guardians against pluripotency and cancer progression. Cell Cycle 2009;8:843-852.

25 Viswanathan SR, Daley GQ, Gregory RI: Selective blockade of microRNA processing by Lin28 Science 2008;320:97-100.

26 Rothe M, Sarma V, Dixit VM, Goeddel DV: TRAF2-mediated activation of NF-kappa B by TNF receptor 2 and CD40 Science 1995;269:1424-1427.

27 Hu H, Chen M, Dai G, Du G, Wang X, He J, Zhao Y, Han D, Cao Y, Zheng Y, Ding D: An Inhibitory Role of Osthole in Rat MSCs Osteogenic Differentiation and Proliferation via Wnt/ $\beta$-Catenin and Erk1/2-MAPK Pathways. Cell Physiol Biochem 2016;38:2375-88.

-28 Xi JC, Zang HY, Guo LX, Xue HB, Liu XD, Bai YB, Ma YZ: The PI3K/AKT cell signaling pathway is involved in regulation of osteoporosis. J Recept Signal Transduct Res 2015;35: 640-645.

-29 Marie PJ: Signaling pathways affecting skeletal health. Curr Osteoporos Rep 2012;10:190-198. 
-30 Chen J, Crawford R, Chen C, Xiao Y: The key regulatory roles of the PI3K/Akt signaling pathway in the functionalities of mesenchymal stem cells and applications in tissue regeneration. Tissue Eng Part B Rev 2013;19:516-528.

-31 Dong K, Hao P, Xu S, Liu S, Zhou W, Yue X, Rausch-Fan X, Liu Z: Alpha-Lipoic Acid Alleviates High-Glucose Suppressed Osteogenic Differentiation of MC3T3-E1 Cells via Antioxidant Effect and PI3K/Akt Signaling Pathway. Cell Physiol Biochem 2017;42:1897-1906.

-32 Vanamee ÉS, Faustman DL: TNFR2: A Novel Target for Cancer Immunotherapy. Trends Mol Med 2017;17:30169-7.

33 Redlich K, Smolen JS: Inflammatory bone loss: pathogenesis and therapeutic intervention. Nat Rev Drug Discov 2012;11:234-250.

-34 Zhang JF, Fu WM, He ML, Xie WD, Lv Q, Wan G, Li G, Wang H, Lu G, Hu X, Jiang S, Li JN, Lin MC, Zhang YO, Kung HF: MiRNA-20a promotes osteogenic differentiation of human mesenchymal stem cells by coregulating BMP signaling. RNA Biol 2011;8:829-838.

35 Jeong BC, Kang IH, Hwang YC, Kim SH, Koh JT: MicroRNA-194 reciprocally stimulates osteogenesis and inhibits adipogenesis via regulating COUP-TFII expression. Cell Death Dis 2014;5:e1532. 